\title{
ANÁLISE DE CUSTOS E LUCRATIVIDADE DA CULTURA DO ALHO
}

Flavio Alberto Oliva, Maycon Vieira Amin, Douglas Fernandes, Ana Paula Verginassi Pocaia, Bruna Coelho de Lima, Layane Oliveira Carvalho, Marcus Ayrton de Lima

Universidade do Oeste Paulista, Faculdade de Ciências Agrárias, Presidente Prudente, São Paulo. e-mail: flavioaoliva@gmail.com

\section{RESUMO}

O alho é uma das culturas mais antigas que se tem conhecimento. Cultivado desde a Antiguidade, o produto é tido por boa parte da população como remédio e, para outros, como excelente condimento pelas propriedades de aroma e sabor. $O$ objetivo do trabalho foi apresentar a mensuração de custos e resultados da produção de alho, subsidiando indicadores necessários para tomada de decisão gerencial e estratégica em propriedades rurais. A metodologia propôs-se a contribuir na organização das informações obtidas em planilhas adequadas possibilitando assim a apuração confiável dos custos e das receitas e, consequentemente, proporcionando informações fidedignas para a estruturação de informações necessárias à gestão da cultura do alho. 0 estudo baseou-se em informações e dados quantitativos dos anos de 2016 e 2017. Como resultado, evidenciou-se a viabilidade econômico-financeira da cultura.

Palavras-chave: Produção, Banana, Custo, Resultado, Financeiro.

\section{COST ANALYSIS AND PROFITABILITY OF GARLIC CULTUREABSTRACT}

\begin{abstract}
Garlic is one of the oldest known cultures. Cultivated since antiquity, the product is considered by most of the population as a remedy and, for others, as an excellent condiment for its aroma and flavor properties. The objective of this study was to present the measurement of costs and results of garlic production, subsidizing indicators necessary for managerial and strategic decision making in rural properties. The methodology proposed to contribute to the organization of the information obtained in appropriate spreadsheets, thus enabling reliable calculation of costs and revenues and, consequently, providing reliable information for the structuring of information necessary for the management of garlic culture. The study was based on information and quantitative data from the years 2016 and 2017. As a result, the economic-financial viability of the crop was evidenced.
\end{abstract}

Keywords: Production, Garlic, Cost, Result, Financial

\section{INTRODUÇÃO}

O alho (Allium sativum L.) é pertencente à família das Alliaceae, a mesma da cebola. Seu porte alterna entre 50 e $70 \mathrm{~cm}$ de altura e suas raízes podem apresentar $50 \mathrm{~cm}$ de profundidade, dependendo da cultivar (EMBRAPA, 1993). Possui folhas estreitas e pontiagudas e o bulbo é fracionado em bulbilhos que ficam agrupados e envoltos por um invólucro de inúmeras túnicas. Se adápta melhor em locais de clima frio e essa condição ideal, proporciona ao vegetal, um florescimento estável e um aumento no teor de substâncias químicas. Em sua composição há, óleos essências; compostos sulfurados; proteínas; carboidratos; vitaminas e sais minerais (MARCHIORI, 2003). 
De acordo com pesquisas feitas por Apolinário et al. (2008), alguns ingredientes ativos presentes no alho, possuem propriedades anticancerígenas, capaz de inibir as nitrosaminas, substâncias nocivas relacionadas ao câncer de estômago, além disso, agem como antibióticos combatendo a bactéria Helicobacter pylori que deteriora a mucosa estomacal propiciando o aparecimento do câncer. Apesar dos benefícios, o consumo excessivo do vegetal pode se tornar tóxico, ocasionando anemia, úlcera gástrica e alergias, entretanto, ainda não há estudos literários que quantifiquem a proporção em que ele se torna nocivo (LEONÊZ, 2008).

O calendário de plantio do alho é de acordo com cada região do Brasil. Nas regiões CentroOeste e Sudeste o vegetal é plantado no mês de março e segue até maio realizando a colheita entre julho e outubro. A região Sul estabelece o plantio de maio a julho e a colheita nos meses de novembro e dezembro (CONAB, 2016).

A hortaliça tem sido cultivada por pequenos e grandes produtores. Segundo a Conab (2017), a produção nacional estimada pelo Instituto Brasileiro de Geografia e Estatística (IBGE) para este ano de 2017 é de 118, 6 mil ton., uma redução de 9,0\% em relação ao ano anterior em que a safra finalizou em 130,4 mil toneladas. Referente à área plantada no país, estima-se 10,8 mil hectares, queda de 3,9\% em comparação ao ano antecessor que obteve área de 11,3 mil hectares.

Os principais estados produtores em 2016 foram Minas Gerais com 15 ton./ha ${ }^{-1}$; Goiás 13,1 ton./ha ${ }^{-1}$; Santa Catarina 10,4 ton./ha ${ }^{-1}$; Rio Grande do Sul 7,9 ton. ha ${ }^{-1}$ e Bahia com 8,9 toneladas por hectare, compondo a produtividade nacional (CONAB, 2017).

\section{METODOLOGIA}

O estudo propôs-se a contribuir na organização das informações obtidas em planilhas adequadas possibilitando assim a apuração confiável dos custos e das receitas e, consequentemente, proporcionando informações fidedignas para a estruturação de informações necessárias à gestão da cultura do alho. O estudo baseou-se em informações e dados quantitativos dos anos de 2016 e 2017, no entanto, possui também caráter qualitativo á medida que se utilizou dessas informações para análises financeiras, econômicas e tomada de decisão.

\section{RESULTADOS}

Para a produção de um hectare de alho, os insumos somam $\mathrm{R} \$ 22.057,80$ (Tabela 1 ), sendo que, o custo com a semente mais o superfosfato simples e o adubo orgânico, representam $64,49 \%$ dos insumos. Os demais, calcário, termofosfato, malathion, sevin, espalhante, palha de arroz, óleo diesel e óleo lubrificante, totalizam 35,51\%.

Tabela 1. Custo dos insumos

\begin{tabular}{|l|l|r|r|r|r|}
\hline \multicolumn{1}{|c|}{ CUSTO DE PRODUC̃O DE 1,00 HECTARE DE ALHO } \\
\hline ESPEIFICAÇÃO & UNIDADE & QUANT. & $\begin{array}{c}\text { CUSTO } \\
\text { UNITÁRIO } \\
\text { R\$ }\end{array}$ & $\begin{array}{c}\text { CUSTO } \\
\text { TOTAL } \\
\text { R\$ }\end{array}$ & $\begin{array}{c}\% \\
\text { INSUMOS }\end{array}$ \\
\hline I - INSUMOS: & & & & & \\
\hline Alho para o plantio & $\mathrm{kg}$ & 900 & 4,00 & $3.600,00$ & $16,32 \%$ \\
\hline Calcário+Frete & $\mathrm{T}$ & 2 & 186,00 & 372,00 & $1,69 \%$ \\
\hline Superfosfato simples & $\mathrm{T}$ & 1 & $5.000,00$ & $5.000,00$ & $22,67 \%$ \\
\hline Termofosfato yoorin BZ-5 & $\mathrm{T}$ & 1 & $3.000,00$ & $3.000,00$ & $13,60 \%$ \\
\hline Adubo orgânico & $\mathrm{m}^{3}$ & 30 & 187,50 & $5.625,00$ & $25,50 \%$ \\
\hline Malathion ou Parathion & Litro & 11 & 100,00 & $1.100,00$ & $4,99 \%$ \\
\hline Sevin 5\% & $\mathrm{kg}$ & 3 & 12,00 & 36,00 & $0,16 \%$ \\
\hline Espalhante adesivo & Litro & 2 & 400,00 & 800,00 & $3,63 \%$ \\
\hline Palha de arroz & $\mathrm{m}^{3}$ & 2 & 40,00 & 80,00 & $0,36 \%$ \\
\hline Óleo diesel & Litro & 800 & 2,96 & $2.368,00$ & $10,74 \%$ \\
\hline Óleo lubrificante & Litro & 20 & 3,84 & 76,80 & $0,35 \%$ \\
\hline Subtotal I & & & & $\mathbf{2 2 . 0 5 7 , 8 0}$ & $\mathbf{1 0 0 , 0 0 \%}$ \\
\hline
\end{tabular}


Com relação ao custo dos serviços, foram contabilizados $\mathrm{R} \$ 35.740,00$ onde a debulha, seleção e desinfecção de bulbilhos mais a limpeza, classificação, preparo e acondicionamento, somam $57,92 \%$, os demais itens, totalizaram $42,08 \%$ da conta de serviços.

Tabela 2. Custo dos Serviços

\begin{tabular}{|l|l|r|r|r|r|}
\hline \multicolumn{1}{|c|}{ II - SERVIÇOS } & UNIDADE & QUANTID. & $\begin{array}{c}\text { CUSTO } \\
\text { UNITÁRIO } \\
\text { R } \$\end{array}$ & $\begin{array}{c}\text { CUSTO } \\
\text { TOTAL } \\
R \$\end{array}$ & $\begin{array}{c}\text { CUSTO } \\
\%\end{array}$ \\
\hline $\begin{array}{l}\text { Debulha, seleção e desinfecção de } \\
\text { bulbilhos }\end{array}$ & $\mathrm{d} / \mathrm{h}$ & 50 & 90,00 & $4.500,00$ & $12,59 \%$ \\
\hline Aração c/ arado reversível & $\mathrm{h} / \mathrm{m}$ & 5 & 100,00 & 500,00 & $1,40 \%$ \\
\hline $\begin{array}{l}\text { Gradagem e incorporação de adubo } \\
\text { orgânico }\end{array}$ & $\mathrm{h} / \mathrm{m}$ & 5 & 100,00 & 500,00 & $1,40 \%$ \\
\hline Marcação de canteiro & $\mathrm{d} / \mathrm{h}$ & 2 & 90,00 & 180,00 & $0,50 \%$ \\
\hline Distribuição de adubo orgânico & $\mathrm{d} / \mathrm{h}$ & 20 & 90,00 & $1.800,00$ & $5,04 \%$ \\
\hline Riscar sulco de plantio & $\mathrm{d} / \mathrm{h}$ & 5 & 90,00 & 450,00 & $1,26 \%$ \\
\hline Adubação química em sulco plantio & $\mathrm{d} / \mathrm{h}$ & 15 & 90,00 & $1.350,00$ & $3,78 \%$ \\
\hline Cobertura palhosa & $\mathrm{d} / \mathrm{h}$ & 25 & 90,00 & $2.250,00$ & $6,30 \%$ \\
\hline Irrigação p/ aspersão & $\mathrm{d} / \mathrm{h}$ & 14 & 90,00 & $1.260,00$ & $3,53 \%$ \\
\hline Pulverizações & $\mathrm{d} / \mathrm{h}$ & 30 & 90,00 & $2.700,00$ & $7,55 \%$ \\
\hline Capinas & $\mathrm{d} / \mathrm{h}$ & 25 & 90,00 & $2.250,00$ & $6,30 \%$ \\
\hline Arranqui e cura inicial & $\mathrm{d} / \mathrm{h}$ & 20 & 90,00 & $1.800,00$ & $5,04 \%$ \\
\hline $\begin{array}{l}\text { Limpeza, classificação, preparo e } \\
\text { acondicionamento }\end{array}$ & $\mathrm{d} / \mathrm{h}$ & 180 & 90,00 & $16.200,00$ & $45,33 \%$ \\
\hline Subtotal II & & & & $\mathbf{3 5 . 7 4 0 , 0 0}$ & $\mathbf{1 0 0 , 0 0 \%}$ \\
\hline
\end{tabular}

Tabela 3. Custo total de produção

\begin{tabular}{|l|r|}
\hline III - OUTROS & \\
\hline Energia Elétrica & $\mathrm{R} \$ 486,00$ \\
\hline Subtotal III & $\mathbf{R} \mathbf{4 8 6 , 0 0}$ \\
\hline TOTAL I + II + III & $\mathbf{R} \mathbf{5 8 . 2 8 3 , 8 0}$ \\
\hline
\end{tabular}

O custo total de produção de um hectare de alho foi calculado em $\mathrm{R} \$ 58.283,80$ (Tabela 3), sendo que $\mathrm{R} \$ 22.057,80$ foram atribuídos a conta de insumos, $R \$ 35.740,00$, a conta de serviços (Tabela 2) e $\mathrm{R} \$ 486,00$ de energia elétrica (Tabela 3 ). Dessa forma, o custo de produção de $1 \mathrm{Kg}$ de alho foi de $\mathrm{R} \$ 11,66$. O custo de produção de uma caixa com $20 \mathrm{Kg}$ foi de $R \$ 233,20$.

Tabela 4. Preço, custo de produção e resultado

\begin{tabular}{|r|r|r|r|r|r|r|r|}
\hline MÊS & Preço/Kg & $\begin{array}{c}\text { Custo/Kg } \\
\mathbf{R} \$\end{array}$ & $\begin{array}{c}\text { Resultado/Kg } \\
\mathbf{R} \$\end{array}$ & $\begin{array}{r}\text { Produção } \\
\text { Mensal(Kg) }\end{array}$ & $\begin{array}{r}\text { Faturamento } \\
\mathbf{R} \$\end{array}$ & $\begin{array}{c}\text { Custo } \\
\mathbf{R} \$\end{array}$ & $\begin{array}{c}\text { Resultado } \\
\mathbf{R} \$\end{array}$ \\
\hline jun/16 & $\mathrm{R} \$ 11,97$ & 11,66 & 0,31 & 1.666 & $19.942,02$ & $19.420,16$ & 521,86 \\
\hline $\mathrm{jul} / 16$ & $\mathrm{R} \$ 12,00$ & 11,66 & 0,34 & 1.666 & $19.992,00$ & $19.420,16$ & 571,84 \\
\hline $\mathrm{ago} / 16$ & $\mathrm{R} \$ 13,33$ & 11,66 & 1,67 & 1.666 & $22.207,78$ & $19.420,16$ & $2.787,62$ \\
\hline $\mathrm{set} / 16$ & $\mathrm{R} \$ 12,47$ & 11,66 & 0,81 & 1.666 & $20.775,02$ & $19.420,16$ & $1.354,86$ \\
\hline $\mathrm{out} / 16$ & $\mathrm{R} \$ 11,73$ & 11,66 & 0,07 & 1.666 & $19.542,18$ & $19.420,16$ & 122,02 \\
\hline $\mathrm{nov} / 16$ & $\mathrm{R} \$ 11,50$ & 11,66 & $-0,16$ & 1.666 & $19.159,00$ & $19.420,16$ & $-261,16$ \\
\hline $\mathrm{dez} / 16$ & $\mathrm{R} \$ 11,50$ & 11,66 & $-0,16$ & 1.666 & $19.159,00$ & $19.420,16$ & $-261,16$ \\
\hline $\mathrm{jan} / 17$ & $\mathrm{R} \$ 13,10$ & 11,66 & 1,44 & 1.666 & $21.824,60$ & $19.420,16$ & $2.404,44$ \\
\hline $\mathrm{fev} / 17$ & $\mathrm{R} \$ 15,28$ & 11,66 & 3,62 & 1.666 & $25.456,48$ & $19.420,16$ & $6.036,32$ \\
\hline $\mathrm{mar} / 17$ & $\mathrm{R} \$ 15,09$ & 11,66 & 3,43 & 1.666 & $25.139,94$ & $19.420,16$ & $5.719,78$ \\
\hline $\mathrm{abr} / 17$ & $\mathrm{R} \$ 15,75$ & 11,66 & 4,09 & 1.666 & $26.239,50$ & $19.420,16$ & $6.819,34$ \\
\hline $\mathrm{mai} / 17$ & $\mathrm{R} \$ 15,99$ & 11,66 & 4,33 & 1.666 & $26.639,34$ & $19.420,16$ & $7.219,18$ \\
\hline & & & & & $266.076,86$ & & $33.034,91$ \\
\hline
\end{tabular}


O alho é uma cultura que sofre os efeitos da sazonalidade, como pode ser observado na Tabela 4, especificamente nos meses de novembro de dezembro, o preço de mercado do produto despencou. Em contrapartida, nos demais meses analisados, o custo de produção esteve abaixo do preço de mercado, proporcionando lucro ao produtor.

\section{DISCUSSÃO}

O conhecimento das condições de mercado e dos recursos naturais dá ao produtor rural os elementos básicos para o desenvolvimento de sua atividade econômica. Cabe agora decidir o que, quanto e como produzir, controlar a ação após iniciar a atividade e por ultimo analisar os resultados alcançados e compará-los com o previsto inicialmente (CREPALDI, 1998).

A análise das planilhas em questão evidencia que, do total do custo de produção $\mathrm{R} \$$ $58.283,80$, os insumos representam $\mathrm{R} \$ 22.057,80$ (37,84\%), os serviços somam $\mathrm{R} \$ 35.740,00$ $(61,32 \%)$ e a energia elétrica $R \$ 486,00(0,84 \%)$. Os insumos, embora com menor participação no custo total, exige atenção para três itens que juntos somam $57,92 \%$ da conta. Em relação aos serviços, dois itens somados representam $57,92 \%$ da conta. Diante do exposto, o produtor de alho deve planejar e atentar para a escolha e opção de preços dos insumos e serviços. Não menos importante, a comercialização do alho requer posicionamento estratégico uma vez que o produto sofre oscilação de preço durante o ano, conforme apresentado na Tabela 4. O período de fevereiro a maio de 2017, apresentou os melhores preços em relação aos demais meses analisados. Concentrar a produção nos meses de melhor preço, proporciona aumento na rentabilidade da atividade. É fundamental entender que a atividade agrícola deve ser planejada e estruturada como qualquer outra atividade, caso contrário, pode se tornar inviável.

\section{CONCLUSÃO}

A análise das planilhas de custos e resultados da cultura do alho, permite concluir que a atividade é viável. A lucratividade para um hectare foi de $12,41 \%$ considerando um faturamento

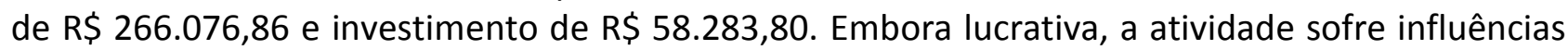
internas e externas em relação a demanda do produto, preço dos insumos e serviços.

\section{REFERENCIAS:}

APOLINÁRIO, A. C. et al. Allium sativum como agente terapêutico para diversas patologias: uma revisão. Revista de Biologia e Farmácia, ISSN 1983-4209, vol. 02, n. 01, 2008.

CONAB. COMPANHIA NACIONAL DE ABASTECIMENTO. Conjuntura Especial Alho, setembro 2016. Disponível em: http://www.conab.gov.br/OlalaCMS/uploads/arquivos/16 $093008 \quad 2027$ alho conjuntura es pecial set 2016.pdf. Acesso em: 5 jul. 2017.

CONAB. COMPANHIA NACIONAL DE ABASTECIMENTO. Conjuntura mensal, janeiro 2017. Disponível em: http://www.conab.gov.br/OlalaCMS/uploads/arquivos/17 $02 \quad 15162859$ alho janeiro 2017.p df. Acesso em: 5 jul. 2017.

CREPALDI, S.A. Contabilidade Gerencial: teoria e pratica. São Paulo: Atlas, 1998.

LEONÊZ, A.C. Alho: alimento e saúde. 30p. Monografia (Especialista em Gastronomia e Saúde) Centro de Excelência em Turismo, Universidade de Brasília (UnB), Brasília, 2008.

MARCHIORI, V. F. Propriedades funcionais do alho (Allium sativum L.). 2003. 
MENEZES SOBRINHO, J. A.; LOPES, C. A.; REIFSHNEIDER, F. J. B.; CHARCHAR, J. M.; CRISÓTOMO, L. A.; CARRIJO, O. A.; BARCOSA, S. A cultura do alho. Brasília: Empresa Brasileira de Pesquisa Agropecuária, Centro Nacional de Pesquisa de Hortaliças. Brasília, 1993. 